\title{
Model Hamiltonians Derived from Kohn-Sham Theory
}

\author{
RENÉ FOURNIER, NAN JIANG \\ Department of Chemistry, York University, Toronto, Ontario, Canada, M3J 1P3
}

Received 20 March 2000; revised 23 May 2000; accepted 6 June 2000

\begin{abstract}
We discuss a general way to derive approximate molecular orbital (MO) methods starting from some reference MO theory. In particular, we present a model Hamiltonian that is based on a Kohn-Sham reference and that is free of adjustable parameters. This Hamiltonian is a linear combination of atom-centered ket-bra operators, each of which is easily derived from the results of Kohn-Sham atomic calculations. The resulting equations are similar to those of extended Hückel $(\mathrm{eH})$ theory and are as efficient computationally as eH. Orbital energies for a few small molecules show that this method is more stable with respect to choice of basis set, and slightly more accurate, than $\mathrm{eH}$. We improved the accuracy of our model Hamiltonian by introducing parameters fitted to the higher level of theory. These parameters define a basis of pseudoatomic orbitals that are, in a certain sense, optimal for the molecule used in the fitting procedure. We illustrate our method by calculating the eigenvalue spectrum of silicon clusters. (C) 2000 John Wiley \& Sons, Inc. Int J Quantum Chem 80: 582-590, 2000
\end{abstract}

Key words: model Hamiltonians; molecular orbitals theory; Kohn-Sham; projectors; tight-binding

\section{Theory}

$\mathrm{f}$ the $n$ lowest eigenvalues $\lambda_{k}$ and associated eigenfunctions $\left|f_{k}\right\rangle$ of an operator $\hat{O}$ are known, the effect of $\hat{O}$ on any function belonging to the $n$-dimensional subspace spanned by $\left\{\left|f_{k}\right\rangle\right\}$ is expressed exactly by a spectral resolution formula [1], $\hat{O}=\sum_{k} \lambda_{k}\left|f_{k}\right\rangle\left\langle f_{k}\right|$. In particular, if we know the atomic orbitals (AO) $\left|\chi_{k}^{\circ}\right\rangle$ and orbital energies $e_{k}^{\circ}$ of $N$

Correspondence to: R. Fournier; e-mail: renef@yorku.ca.

Contract grant sponsors: Natural Sciences and Engineering Research Council of Canada; York University. atoms $A, B, C, \ldots$, within, say, Kohn-Sham (KS) theory using some exchange-correlation potential $V_{\mathrm{xc}}$, then we can write the Hamiltonian of $N$ infinitely separated atoms $A, B, C, \ldots$, within the same model as $\hat{H}=\sum_{k} e_{k}^{\circ}\left|\chi_{k}^{\circ}\right\rangle\left\langle\chi_{k}^{\circ}\right|$, where the sum is over all $\mathrm{AO}$ on all atoms.

Electronic structure calculations on molecules can be done using a variety of basis sets: AO, atomcentered Cartesian Gaussians or numerical Slatertype orbitals, plane waves, etc. If we leave aside considerations about numerical efficiency or ease of implementation, the $\mathrm{AO}$ basis stands out as a natural choice; it is the starting point of the linear combination of AO (LCAO) theory of chemical 
bonding. We can view $\mathrm{AO}$ bases for molecular calculations as made up of the $n$ lowest energy $\mathrm{AO}$ of infinitely separated atoms $\left\{\left|\chi_{k}^{\circ}\right\rangle\right\}$ translated in space to the positions nuclei have in the molecule to give a basis set that we denote by $\left\{\left|\chi_{k}\right\rangle\right\}$. A first-principles calculation would then proceed with that finite basis and the exact Hamiltonian. If instead we construct a model Hamiltonian $\hat{H}^{(m)}$ from the $\left|\chi_{k}^{\circ}\right\rangle$ by translation in space, in a way similar to how the AO basis set is constructed, we get $\hat{H}^{(m)}=\sum_{k} e_{k}^{\circ}\left|\chi_{k}\right\rangle\left\langle\chi_{k}\right|$. Defining the metric matrix $\left\{S_{i j}\right\}$ over normalized AO through $S_{i i}=1$ and $S_{i j}=\left\langle\chi_{i} \mid \chi_{j}\right\rangle$, the matrix elements of $\hat{H}^{(m)}$ are

$$
\begin{aligned}
& \hat{H}_{i i}^{(m)}=e_{i}^{\circ}+\sum_{k \neq i} e_{k}^{\circ} S_{i k}^{2} \\
& \hat{H}_{i j}^{(m)}=\sum_{k} S_{i k} e_{k}^{\circ} S_{k j}, \quad i \neq j .
\end{aligned}
$$

An equation similar to Eq. (1) was proposed by Newton et al., as an "intuitively reasonable formula" [2]. Newton et al. noted that their equation satisfied some symmetry requirements, but they did not obtain it from first principles, and they introduced adjustable parameters in defining their nonempirical molecular orbital (NEMO) method. Linderberg and Öhrn proposed the Hamiltonian [Eq. (1)] and used it to illustrate how electron binding energies can be calculated using a Green's function [3]. Shortly after, model Hamiltonians of the form of Eq. (1) were used [under the name "modified extended Hückel" $(\mathrm{eH})]$ to calculate the photoelectron spectra of azines [4] and nuclear quadrupole coupling constants of some oxy-compounds of bromine and chlorine [5]. A more detailed discussion of Eq. (1) was given by Linderberg et al. who coined the term energy weighted maximum overlap (EWMO) to describe their method [6]. Linderberg et al. applied EWMO to electron spin resonance problems $[7,8]$ and particle-hole excitations [9]. The EWMO method is not as popular as eH or other semiempirical methods, but we think that it is very important for theory and deserves a close look. It has no adjustable parameter, and it can be used to approximate any first-principles molecular orbital (MO) theory, including KS theory. Moreover, if we write Eq. (1) in matrix form, $\mathbf{H}=$ SeS, we see that $\mathbf{e}$ is a diagonal matrix of $\mathrm{AO}$ energies and that better model Hamiltonians may be obtained by adding empirical off-diagonal elements to e. On the other hand, if we neglect all the terms in the sum of Eq. (1) except those with $k=i$ and $k=j$, an approximation that seems justified when $\mathrm{AO}$ on different atoms overlap only weakly, we get

$$
\begin{aligned}
& \hat{H}_{i i}^{(m)}=e_{i}^{\circ} \\
& \hat{H}_{i j}^{(m)}=\left(e_{i}^{\circ}+e_{j}^{\circ}\right) S_{i j}, \quad i \neq j .
\end{aligned}
$$

This is the well-known Wolfsberg-Helmholtz formula [10] of eH theory [11, 12], with the parameter $K$ set equal to 2 . This way to arrive at $\mathrm{eH}$ theory is interesting, but it is rarely mentioned. (However, Linderberg and Öhrn did mention the similarity between Eqs. (1) and (2) [3].) If we retain all terms in the sum in Eq. (1) we might improve over eH while keeping a very efficient method. But more importantly, we can generalize the model Hamiltonian [Eq. (1)] in two ways.

First, we can take $\hat{H}^{(m)}=\sum_{k} v_{k}^{\circ}\left|\phi_{k}\right\rangle\left\langle\phi_{k}\right|$ where $v_{k}^{\circ}$ and $\left|\phi_{k}^{\circ}\right\rangle$ are eigenvalues and eigenfunctions of modified atomic problems, or, pseudoatom orbitals (PAO). Porezag et al. [13] proposed an analogous ideaa basis of PAO obtained by solving the KS atomic problem in a constraining spherical potential-in the context of tight-binding (TB) methods. These authors reported that TB calculations with a nonself-consistent potential gave better results when the basis functions and potential are derived from a superposition of PAO instead of a superposition of free atom $\mathrm{AO}$. We surmise that the use of some kind of modified AO (maybe the PAO of Porezag et al.) would improve the accuracy of Eq. (1) as well.

Second, we note that if we knew the solution to the molecular problem in the AO basis, the KS Hamiltonian could be written as $\hat{H}=\sum_{i} \epsilon_{i}\left|\psi_{i}\right\rangle\left\langle\psi_{i}\right|=$ $\sum_{i} \epsilon_{i} \sum_{a, b} C_{i a}\left|\chi_{a}\right\rangle C_{i b}^{*}\left\langle\chi_{b}\right|$, or

$$
\hat{H}=\sum_{a, b}\left|\chi_{a}\right\rangle W_{a b}\left\langle\chi_{b}\right|,
$$

where $\left|\psi_{i}\right\rangle$ is a molecular orbital, $\epsilon_{i}$ is an MO energy, $\left|\chi_{a}\right\rangle$ is an AO, $C_{i a}$ is the coefficient of $\mathrm{AO}$ " $a$ " in $\mathrm{MO}$ " $i$," and $W_{a b} \equiv \sum_{i} \epsilon_{i} C_{i a} C_{i b}^{*}$ can be viewed as a weighted sum of MO energies for the $a b$ pair of AO. In calculations it is practical to view $W_{a b}$ as defined by the matrix equation $\mathbf{H}^{(\mathbf{m})}=\mathbf{S W S}$ (or, $\mathbf{W}=$ $\left.\mathbf{S}^{-1} \mathbf{H}^{(\mathbf{m})} \mathbf{S}^{-1}\right)$. Expansion of operators in a basis, as in Eq. (3), is well-known in the general theory [1]. We merely wish to point out here that Eq. (3) is a very promising starting point for devising model Hamiltonians by modeling the matrix $\left\{W_{a b}\right\}$ in a basis of AO. Indeed, even the crudest model, $W_{a b}=\delta_{a b} e_{a}^{\circ}$, is useful as it brings us back to Eq. (1) and, with a further approximation, to eH theory. Nothing that simple works as well when trying to model directly 
the Hamiltonian matrix $\left\{H_{i j}\right\}$, as done in most TB methods [14].

KS theory can, in principle, yield exact groundstate properties in an $\mathrm{MO}$ formalism. Therefore, it can be viewed as the correct underlying theory for all variants of $\mathrm{eH}$ and $\mathrm{TB}$ schemes, including EWMO and its generalization [Eq. (3)]. Lindholm and Lundqvist gave a similar argument for interpreting the semiempirical theories PPP, MINDO, $\mathrm{MNDO}, \mathrm{CNDO} / \mathrm{S}$, SPINDO, and HAM as approximations to KS theory [15]. They showed that the KS energy expression, with exact exchange and correlation contributions, can be put in the same form as that of semiempirical theories. Lindholm and Åsbrink used this conceptual connection as a guide in approximating different terms of the energy in HAM, but they fitted parameters of their method to experiment instead of taking them from KS calculations [16].

Before discussing the results of calculations, we want to indicate the relation between our approach and previous work. Our Eq. (1) is the same as for EWMO [3, 6], although we propose it on different grounds-by making an analogy between the expansion of $\mathrm{MO}$ by $\mathrm{LCAO}$, and the approximate representation of operators by linear combination of atomic orbital operators. Differences with EWMO are that we recommend to generalize Eq. (1) by using PAO or Eq. (3); we interpret Eqs. (1) and (3) as approximate KS Hamiltonians; and, in order to be consistent with this interpretation, we choose to parametrize Eqs. (1) and (3) directly from reference KS calculations. Our goal is to derive an efficient semiempirical theory from KS density functional theory (DFT), the same goal as in the HAM method [16]. But our approach differs greatly from HAM in the details: it is much simpler, it does not require any fit to (or, input from) experiment, and its focus is on one-electron energies instead of the total energy. A disadvantage of our approach, compared to HAM, is that we do not specify how to calculate the total energy [see Eq. (5)].

\section{Tests on Small Molecules}

We calculated the orbital energies of the molecules $\mathrm{H}_{2} \mathrm{O}, \mathrm{CH}_{4}, \mathrm{~N}_{2}, \mathrm{C}_{2} \mathrm{H}_{4}$, and $\mathrm{CO}_{2}$ with different methods. First, we used KS in the local spin density (LSD) approximation with the Vosko-Wilk-Nusair parametrization for the exchange-correlation potential and $(4 s, 3 p, 1 d)$ basis sets on $\mathrm{C}, \mathrm{N}$, and $\mathrm{O}$, and a $(3 s, 1 p)$ basis on $\mathrm{H}$. This is our reference: All other methods use AO calculated in this approximation and aim to reproduce its results for molecules. The other methods are non-self-consistent. They simply require diagonalization of the matrix $\left(\mathbf{S}^{-1 / 2} \mathbf{H}^{(\mathbf{m})} \mathbf{S}^{-1 / 2}\right)$ where $\mathbf{H}^{(\mathbf{m})}$ is given by either: (1) Eq. (1); (2) Eq. (2) with the right side multiplied by 0.875 (eH with $K=1.75$ ); or (3) Eq. (2) (eH with $K=2$ ). We made variations on these methods by choosing different subsets of the atomic KS-DFT eigenfunctions: (a) minimal set of valence $\mathrm{AO}$; (b) minimal set of $\mathrm{AO}$ that includes core orbitals; (c) the full set of atomic eigenfunctions. The results are given in Table I. We point out that Stowasser and Hoffmann recently discussed the chemical meaning of $\mathrm{KS}$ and eH eigenvalues [17]. However, the standard eH model they used is defined from Slater $\mathrm{AO}$ and ionization potentials, instead of KS AO and eigenvalues, and therefore their comparison of eH and KS molecular orbital energies is not as straightforward as it is here.

The best model in principle is 1(c). The mean absolute deviation (MAD), in electron volts, between KS and 1(c) orbital energies [valence, lowest unoccupied molecular orbitals (LUMO), and LUMO+1 in some cases] are: 1.4 for $\mathrm{H}_{2} \mathrm{O}, 2.3$ for $\mathrm{CH}_{4}, 2.1$ for $\mathrm{C}_{2} \mathrm{H}_{4}, 1.4$ for $\mathrm{N}_{2}$, and 1.7 for $\mathrm{CO}_{2}$. The best of the four eH models, 2(a), gives MAD for these molecules (in the same order) of 4.4, 7.7, 1.8, 7.5 and 4.4; these go down to $1.7,3.4,1.6,2.5$, and 2.2, respectively, if we ignore the highest energy $\mathrm{MO}$, which are very poorly predicted by eH (except in $\mathrm{C}_{2} \mathrm{H}_{4}$ ) . Table I shows that models 1(c) and 2(a) get a wrong order for some orbitals, and although it cannot be seen from Table I, model 2(a) gets the wrong orbital as the LUMO of $\mathrm{H}_{2} \mathrm{O}$. Model 1(c) gives the smallest MAD overall, and the MAD for models 1(a), $1(b)$, and 1(c) decrease in that order, as one would expect when increasing the size of the basis and improving the description of the Hamiltonian. Going from 1(a) to 1(c) shifts the eigenvalues to higher energies, which particularly improves the position of the lowest valence orbital (except for $\mathrm{CO}_{2}$ ) and of the LUMO. By way of contrast, the eH model gets worse when the basis gets larger, that is, in going from 2(a) to 2(b) and 2(c). [We simply list the $n$ lowest eigenvalues of model 2(c) in increasing order; that order is generally not consistent with KS and other calculations.] Actually, 2(c) is seriously flawed because the proportionality of $H_{i j}$ and $S_{i j}$ implied by Eq. (2) holds only when the effect of the operator is nearly constant across the region of overlap. $\left(C_{i j}\right.$ and $S_{i j}$ are clearly proportional when the operator $\hat{C}$ is a constant $c$, in which case $C_{i j}=c S_{i j}$.) 
TABLE I

Orbital energies (eV) of $\mathrm{H}_{2} \mathrm{O}, \mathrm{CH}_{4}, \mathrm{C}_{2} \mathrm{H}_{4}, \mathrm{~N}_{2}$, and $\mathrm{CO}_{2}$, degeneracy in parentheses.

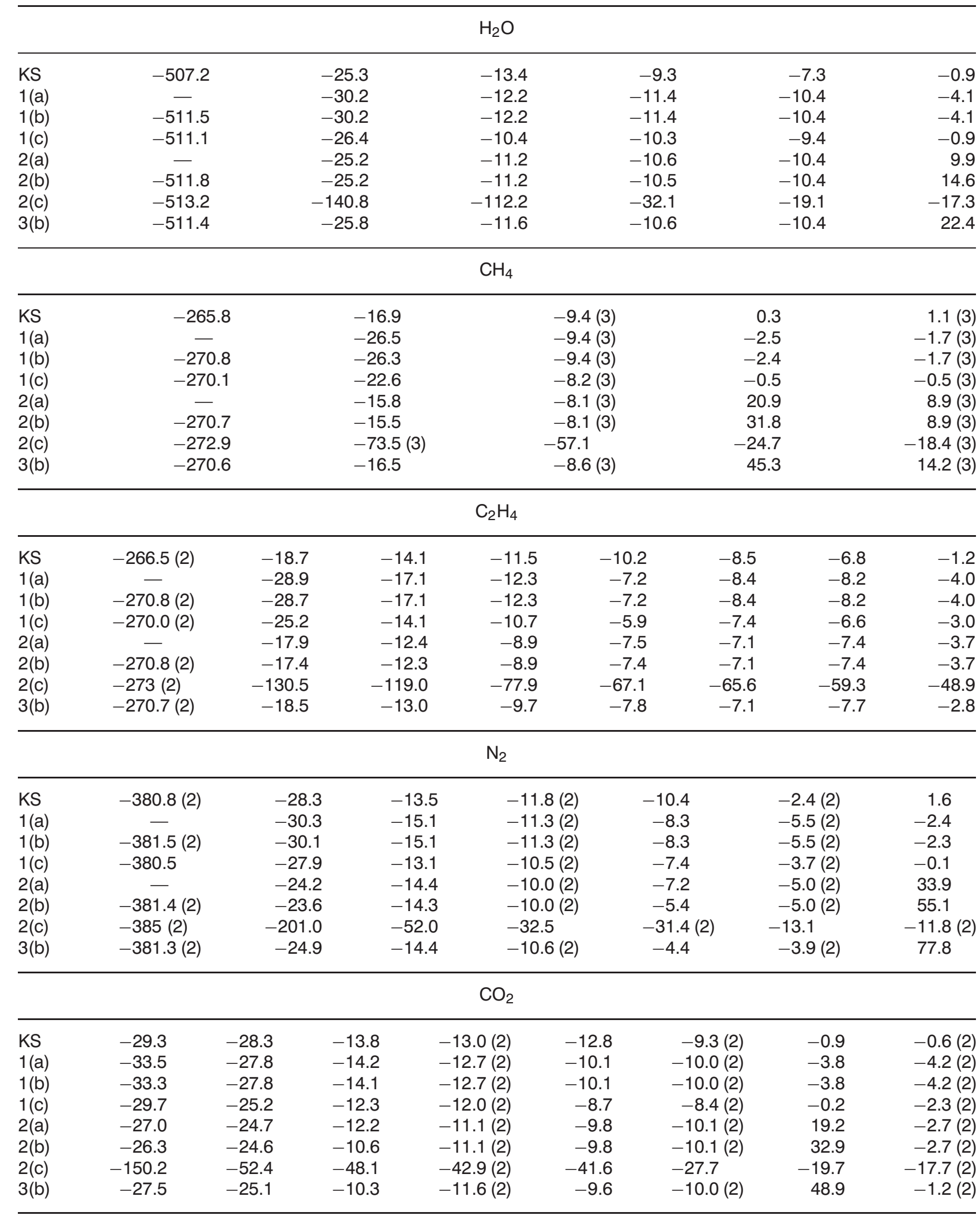


Equation (2) fails for core-valence matrix elements because the potential (and effective Hamiltonian) varies rapidly near the nuclei. It also fails for matrix elements involving diffuse functions because, then, functions overlap over a large region of space, and it is unrealistic to assume that the one-electron effective Hamiltonian is constant over that region of space. The latter problem can be very serious for metals such as sodium or silver where the description of interactions mediated by outer $s \mathrm{AO}$ is crucial. Therefore, we expect Eq. (1) to be better than Eq. (2) for describing bonding in metals. Changing $K$ from 1.75 to the theoretically more correct value of 2.00 [model 3(b)] generally does not improve eH models. On the contrary, $K=2.00$ is optimal for Eq. (1): Results deteriorate if we multiply the righthand side of Eq. (1) by a factor different from 1. In other words, if we introduced an adjustable factor in Eq. (1) and fitted it to KS eigenvalues, we would get something very close to the theoretically correct value. The highest occupied molecular orbital (HOMO)-LUMO gap is a critical property for chemical reactivity, electronic spectroscopy, and many properties. Table I shows that HOMO-LUMO gaps calculated by 1(c) are not very good, but they are somewhat better than those of 2(a). Taken together, our results illustrate that $\mathrm{eH}$ is essentially a minimal valence basis theory that cannot be improved in a straightforward way, whereas model 1 improves with basis set size.

\section{Tests on Silicon Clusters}

One of the possible applications of a model Hamiltonian such as Eq. (3) are in studying the electronic structure of clusters. Finite-temperature and dynamic properties of small clusters require large numbers of energy evaluation; realistic models of materials often require large clusters with thousands of atoms. Both types of problems require very efficient computational methods. We tested model Hamiltonians by comparison to KS-DFT for two isomers of $\mathrm{Si}_{4}$ and $\mathrm{Si}_{7}$ and cluster fragments of the Si bulk diamond structure shown in Figure 1. Our KS-DFT calculations use a model core potential and treat explicitly only the four valence electrons with a (311/311/1) Gaussian basis set. We did two series of KS calculations: with a standard LSD potential (that of Vosko, Wilk, and Nusair) and with a standard gradient-corrected functional (Becke's 1988 exchange with Perdew and Wang 1986 correlation). The two exchange-correlation functionals give nearly identical eigenvalues for the silicon clusters we looked at, so we report only the LSD results here. In Figure 2 we display eigenvalue spectra as "densities of states" (DOS)—superpositions of Gaussians with a width of $0.2 \mathrm{eV}-$ for $\mathrm{Si}_{4}$ rhombus and linear isomers, and for $\mathrm{Si}_{7}$ pentagonal bipyramid and tricapped tetrahedron isomers, calculated by KS-DFT, by Eq. (1) ("diagonal W" in Fig. 2), and by Eq. (4) (see next section and "PAO" in Fig. 2). There is a rough correspondence between KS-DFT and Eq. (1) in the positions of peaks and eigenvalue degeneracy, but the agreement is poor. The MAD between the KS-DFT eigenvalues of the rhombus and linear $\mathrm{Si}_{4}$ is $0.6 \mathrm{eV}$, whereas the MAD between KS-DFT and Eq. (1) are $1.0 \mathrm{eV}$ for the rhombus and $0.8 \mathrm{eV}$ for the linear form. Therefore, Eq. (1) would not be good enough to predict or explain differences in the photoelectron spectra of two very different cluster isomers. The same can be said about $\mathrm{Si}_{7}$ (Fig. 2). We also looked at the DOS of $\mathrm{Si}_{29}$ and $\mathrm{Si}_{35}$ bulk silicon fragments. They are not very informative and are not shown here. What we learn from them can be summed up in few words: The mean and width of the energy band are reasonably well reproduced by the model Hamiltonian [Eq. (1)], but KS-DFT and Eq. (1) differ greatly in the detailsthe match between the two is poor. As another test, we compared DOS for different $\mathrm{Si}_{28}$ clusters obtained by removing one atom from the $\mathrm{Si}_{29}$ cluster of Figure 1. We considered removal of the central atom (" $\left.4 c^{\prime \prime}\right)$, one of the 4-coordinated atoms bonded to the central one (" 4 "), one of the 3-coordinated atoms bonded to atom " 4 " (" 3 "), and one of the 2coordinated atoms bonded to atom " 3 " ("2"). The difference in DOS relative to $\mathrm{Si}_{29}$ are quite complicated and do not yield much insight, so we do not show them here. Instead, we compare DOS two at a time, by computing a measure of mismatch $\Delta$ (DOS), which we define (up to an arbitrary constant) as $\Delta(A, B)=\int\left[\operatorname{DOS}_{A}(\epsilon)-\operatorname{DOS}_{B}(\epsilon)\right]^{2} d \epsilon$. The values of $\Delta(\mathrm{KS} / 4 \mathrm{c}, \mathrm{KS} / n)(n=4,3,2)$ are 2.3, 2.9, and 3.2, respectively. The corresponding values for $\Delta$ [Eq. (1) $/ 4$ c, Eq. $(1) / n](n=4,3,2)$ are 2.0, 2.1, and 2.2. More importantly for comparing KS and Eq. (1), the $\Delta[\mathrm{KS} / n$, Eq. $(1) / n](n=4 c, 4,3,2)$ vary between 5.1 and 5.4. We conclude that Eq. (1) is not accurate enough to describe reliably the change in electronic structure associated with a missing atom defect. We did eH calculations for several of these clusters and found that it is not significantly better or worse than Eq. (1).

Overall, our assessment of the model Hamiltonian [Eq. (1)] is that it gives a qualitatively correct 

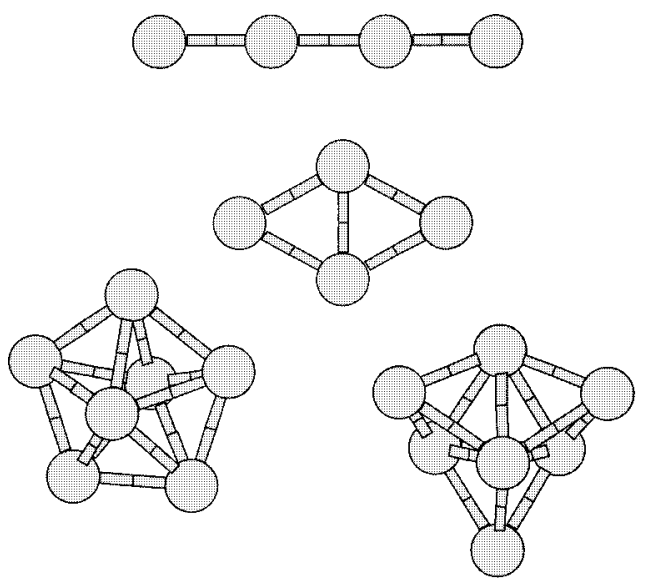

(a)

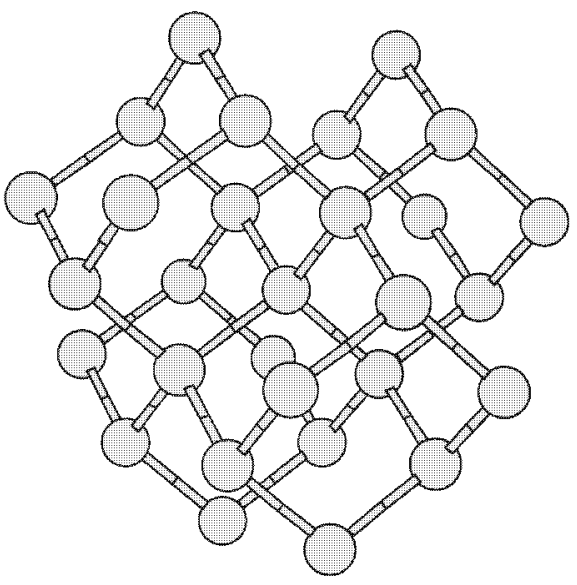

(c)

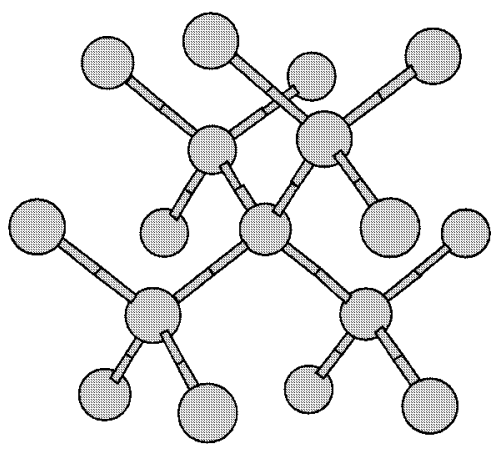

(b)

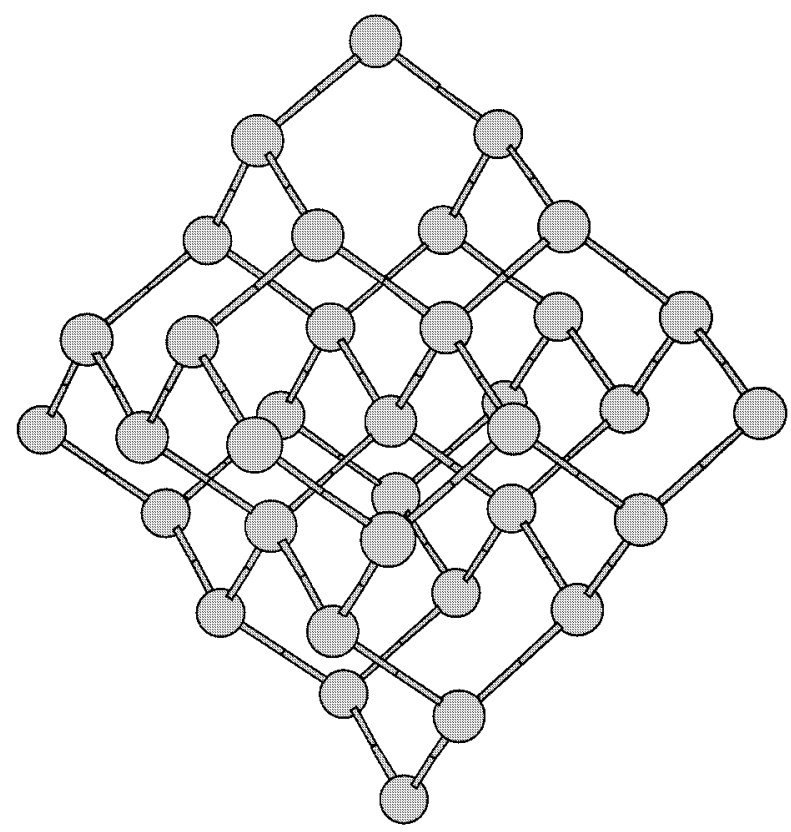

(d)

FIGURE 1. Silicon clusters used in testing model Hamiltonians.

description of $\mathrm{MO}$ symmetry and energy ordering as does eH theory. It has a better theoretical basis than $\mathrm{eH}$ theory, and it affords a more robust and slightly more accurate method for calculating $\mathrm{MO}$ energies than eH. However, it gives only low accuracy: Errors of 1-2 eV on $\mathrm{MO}$ energies are typical. It is not accurate enough to describe subtle changes in electronic structure associated with changes in geometry or defects. But Eq. (1) is only a starting point, a rough approximation to Eq. (3). We describe a better model for the matrix $\left\{W_{a b}\right\}$ in the next section.

\section{W Parametrized to Give Pseudoatomic Orbitals}

As a first step beyond Eq. (1) we tried the following model:

$$
\begin{array}{cl}
W_{a b}=e_{a}^{\circ}+\Delta(n, \ell) & \text { when } a=b, \\
=K\left(n, \ell, n^{\prime}, \ell^{\prime}\right) & \text { when } a \neq b, A=B, \\
& \quad \ell=\ell^{\prime}, m=m^{\prime}, \\
=0 & \text { otherwise. }
\end{array}
$$




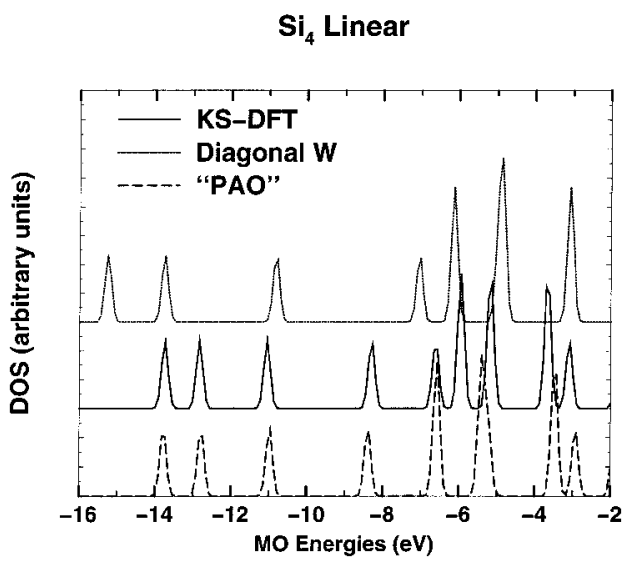

(a)

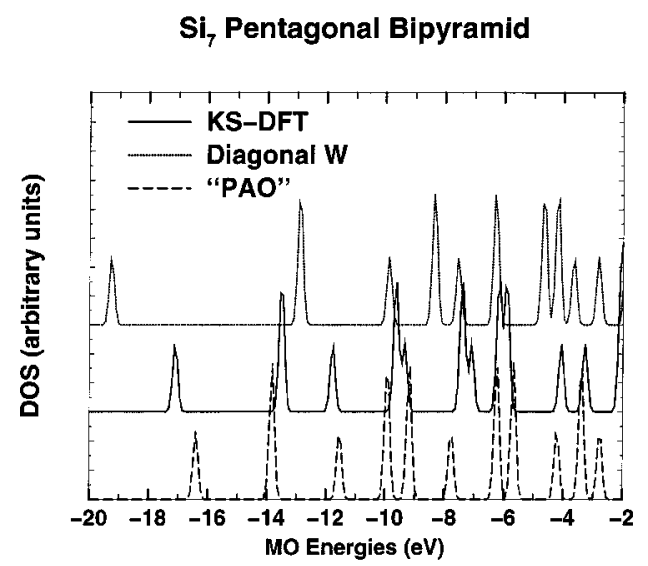

(c)

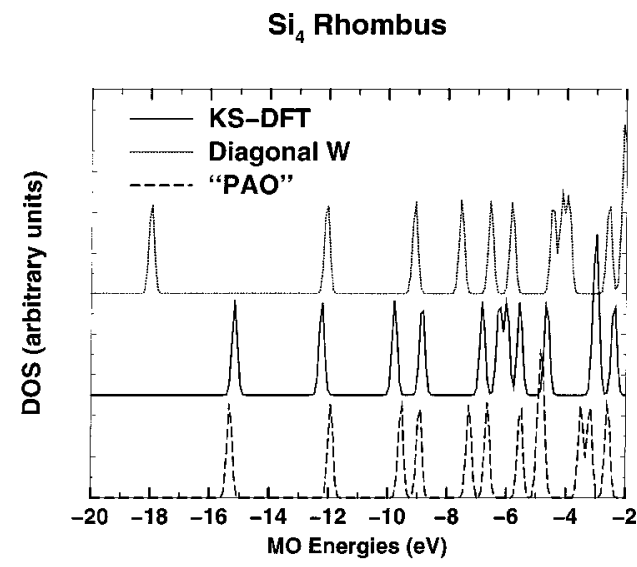

(b)

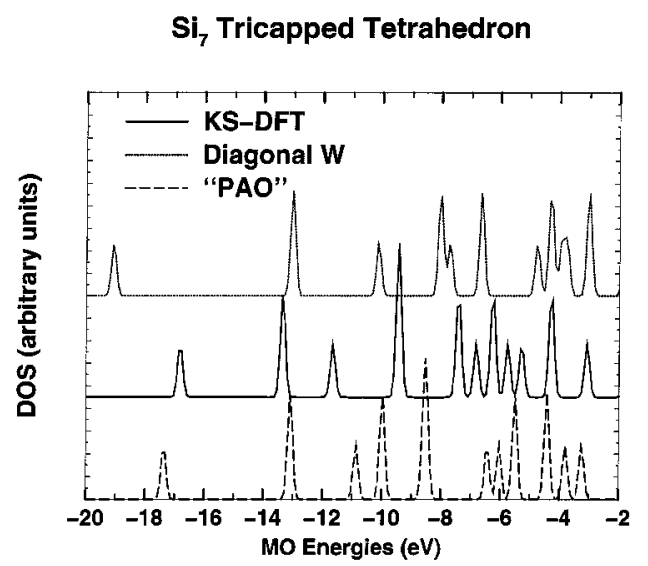

(d)

FIGURE 2. Densities of states (DOS) obtained by Gaussian broadening ( $0.2 \mathrm{eV}$ width) of the discrete eigenvalue spectrum of small silicon clusters.

Here, $A$ and $B$ label atoms, $a$ and $b$ label $\mathrm{AO}$ functions, $n$ and $\ell\left(n^{\prime}, \ell^{\prime}\right)$ label atomic subshells $(3 s, 3 p, 4 s, \ldots)$, and $m\left(m^{\prime}\right)$ label components of a subshell (e.g., different $m$ for $\left.p_{x}, p_{y}, p_{z}\right) ; \Delta(n, \ell)$ shift $\mathrm{AO}$ energies, whereas $K\left(n, \ell, n^{\prime}, \ell^{\prime}\right)$ mix $\mathrm{AO}$ of the same symmetry from different shells. The parameters were allowed to vary in a range from -5 to $+5 \mathrm{eV}$ and were optimized by a genetic algorithm minimization of the root mean square (RMS) deviation relative to the KS-DFT eigenvalues of some reference silicon cluster. In the limit of separated atoms, the exact $\left\{W_{a b}\right\}$ is diagonal with elements equal to $e_{a}^{\circ}$ : all $\Delta(n, \ell)$ and $K\left(n, \ell, n^{\prime}, \ell^{\prime}\right)$ are zero. The parameters $\Delta(n, \ell)$ and $K\left(n, \ell, n^{\prime}, \ell^{\prime}\right)$ act in a way to produce a sort of spherically symmetric excited state of the atom that is (in a sense) optimal for the molecule. Therefore, this parametrization of $\left\{W_{a b}\right\}$ is almost equivalent to using PAO and Eq. (1).
PAO can be obtained by diagonalizing a block of $\left\{W_{a b}\right\}$ corresponding to a single atom. For example, when we parametrize $\left\{W_{a b}\right\}$ using the linear $\mathrm{Si}_{4}$ as the reference, we find that the two lowest energy PAO can be characterized as: a $\widetilde{3 s}$ that is $98 \% 3 s$ and $2 \%$ higher $s$; and a $\widetilde{3 p}$ that is $99.8 \% 3 p$, and $0.2 \%$ higher $p$. For rhombic $\mathrm{Si}_{4}$, the lowest energy PAO are: a $\tilde{3 s}$ that is $99.5 \% 3 s$ and $0.5 \%$ higher $s$; and a $\tilde{3 p}$ that is $85 \% 3 p$, and $15 \%$ higher $p$. In principle, these PAO could be obtained by solving the KS-DFT problem for an atom in a suitably chosen spherically symmetric external potential. That would be similar to the PAO of Porezag et al. [13]. But, contrary to Porezag et al., we fit directly to MO energies of reference KS-DFT calculations and do not try to find the external potential that would give rise to a given PAO. Clearly, we obtain different PAO depending on the 
cluster (or atoms) we choose for fitting. Thus, for applications to silicon materials, it will be important to obtain a few representative PAOs for silicon atoms in 2-, 3-, or 4-coordinated sites of $\mathrm{Si}$ diamond fragment clusters such as those of Figure 1. (In order to apply Eq. (4) to arbitrary silicon structures, one would have to describe how parameters change with geometry. This may be difficult, but since our model Hamiltonians have a close connection to some KS-DFT reference, they could at least be used to interpolate in the neighborhood of structures for which KS-DFT results are known.)

We went in a stepwise way from the model of Eq. (1) to that of Eq. (4): First, we show the results of Eq. (1) $[W(0)]$; then, we allow the diagonal terms of $W$ corresponding to the $3 s$ and $3 p$ to shift relative to the AO energies [ $W(s p)]$; then, we allow all diagonal terms of $W$ to shift [W(ii)]; next, we introduce off-diagonal terms that mix the s-symmetry $\mathrm{AO}$ $[W(i j, s)]$; and finally, we allow mixing within the $p$-symmetry AO $[W(i j, s p)]$. At each step, all parameters are free to change and get reoptimized, so that the final result is probably very close to the global minimum in parameter space. Table II summarizes the results. For simplicity, we did not attempt to match MO from different calculations on criteria other than energy: We report a simple RMS deviation between two sets of numbers (the eigenvalues). However, it is clear that there are some MO energy ordering mismatch between calculations, therefore the RMS that we report here should be viewed as lower bounds. For example, linear $\mathrm{Si}_{4}$ in model $W(0)$ has an RMS of 0.95 (instead of 0.87 ) if we match MO of KS-DFT with those of Eq. (1) according to their symmetry (and energy). We expect that the true RMS in the other cases are also roughly $0.1 \mathrm{eV}$ higher than those in Table II. Judging simply from RMS deviations, Eq. (1) gets progressively worse

\section{TABLE II}

RMS deviation $(\mathrm{eV})$ between eigenvalues calculated by KS-DFT, and by Eq. (4) with different models for $W$.

\begin{tabular}{lcccc}
\hline Model $^{\mathrm{a}}$ & Linear Si $_{4}$ & Rhombic Si $_{4}$ & $\mathrm{PBP} \mathrm{Si}_{7}$ & $\mathrm{TT} \mathrm{Si}_{7}$ \\
\hline$W(0)$ & 0.87 & 1.11 & 1.53 & 1.41 \\
$W(s p)$ & 0.68 & 0.55 & 0.99 & 0.80 \\
$W(i i)$ & 0.48 & 0.42 & 0.86 & 0.67 \\
$W(i j, s)$ & 0.26 & 0.37 & 0.72 & 0.56 \\
$W(i j, s p)$ & 0.25 & 0.36 & 0.69 & 0.54 \\
\hline
\end{tabular}

a See text for the meaning of the symbols. from linear $\mathrm{Si}_{4}$, to rhombic $\mathrm{Si}_{4}$, to $\mathrm{Si}_{7}$. This can be understood in terms of coordination numbers: zero for separated atoms where Eq. (1) is exact, one or two in linear $\mathrm{Si}_{4}$, two or three in rhombic $\mathrm{Si}_{4}$, and four or more in both $\mathrm{Si}_{7}$ isomers. Likewise, the optimal parameters generally get further from zero in the same order, although this is not systematic. The most important parameter, the energy shift of the 3s, is $1.02 \mathrm{eV}$ for linear $\mathrm{Si}_{4}, 3.07 \mathrm{eV}$ for rhombic $\mathrm{Si}_{4}$, $2.49 \mathrm{eV}$ for pentagonal bipyramid $\mathrm{Si}_{7}$, and $2.68 \mathrm{eV}$ for tricapped tetrahedral $\mathrm{Si}_{7}$. Model Eq. (4) cuts the RMS deviation considerably: by 0.6 to $0.8 \mathrm{eV}$, or, by a factor between 2.0 and 3.5. Figure 2 shows how the agreement with KS-DFT improves in going from Eq. (1) to (4). This is noteworthy considering that Eq. (4) is still a rather crude attempt at modeling $\left\{W_{a b}\right\}$-it ignores differences between atoms in a cluster, and all interatomic terms. Using different PAO on different atoms will not be very difficult to implement, and it may also be possible to improve accuracy with a simple model for interatomic terms in $\left\{W_{a b}\right\}$. But, on the other hand, in practical applications of Eq. (3) one will not know the KS results in advance, and, thus, the RMS deviations of Table II are lower bounds to what one could get by a priori modelling of $\left\{W_{a b}\right\}$ with Eq. (4).

Finally, we want to point out that the PAO obtained as we described could find other uses than for constructing approximate model Hamiltonians. A small set of the lowest energy PAO defines a compact basis set, which might be useful in firstprinciples methods. We believe that a minimal basis of PAO would generally give better results in molecular calculations than a minimal basis of AO. But this remains to be verified by calculations.

\section{Concluding Remarks}

One can go in a systematic way from a firstprinciples one-electron effective Hamiltonian to more approximate methods. Equation (1) gives eigenvalues in better agreement with the underlying model (here, KS-DFT) than eH, and it can be improved through either the use of PAO, or, of a model $W$ [Eq. (3)] in place of the diagonal matrix of $\mathrm{AO}$ energies. It is not necessary to invoke any experimental result: One can parametrize $W$ entirely from a higher level of theory in a way similar to TB models [14]. Likewise, there is a well-known and straightforward way to calculate approximate KSDFT total energies. One defines 2-body potentials for 
atoms of type $A$ and $B U\left(R_{A B}\right)$ through

$$
U\left(R_{A B}\right)=E_{\mathrm{KS}}\left(R_{A B}\right)-\Delta\left(\sum_{i}^{\text {occ. }} n_{i} \epsilon_{i}\right),
$$

where $E_{\mathrm{KS}}\left(R_{A B}\right)$ is the energy, relative to separated fragments, of a reference system with interatomic distance $R_{A B}$ calculated accurately by KS-DFT, and $\Delta\left(\sum_{i}^{\text {occ. }} n_{i} \epsilon_{i}\right)$ is the difference in the sum of occupied orbital energies between the separated and interacting fragments calculated with the model Hamiltonian.

$\mathrm{KS}$ theory can in principle give exact results for the ground state of molecules within a MO formalism [18] and, in practice, it gives rather good results. Therefore, it seems possible to derive very efficient and fairly accurate methods from KS theory. Different arguments for this were put forth by Lindholm and Lundqvist [15], by Harris [19], and by Foulkes and Haydock [20]. The way we arrive at Eqs. (1)-(3) illustrates that the spectral resolution formula is a natural approach to semiempirical theories. This should not come as a surprise considering that projectors afford a very elegant formulation of quantum theory [1]. We hasten to add that there already is a conceptual bridge between first-principles and semiempirical theories $[15,21]$, and that projectors have already proven very useful in approximate methods [22]. But we feel that it is worthwhile to make a more systematic use of projectors [Eq. (3)] in the context of approximate KS-DFT, and we plan to do this in the future.

\section{ACKNOWLEDGMENTS}

One of us (R.F.) is grateful to Jan Linderberg for providing reprints and for informative discussions about the EWMO method. This work was supported by the Natural Sciences and Engineering Research Council of Canada and a grant from York University.

\section{References}

1. Löwdin, P.-O. Linear Algebra for Quantum Theory; Wiley: Toronto, 1998.

2. Newton, M. D.; Boer, F. P.; Lipscomb, W. N. J Am Chem Soc 1966, 88, 2353.

3. Linderberg, J.; Öhrn, Y. Propagators in Quantum Chemistry; Academic: New York, 1973; pp. 81-83.

4. Spanget-Larsen, J. J Electron Spectrosc Related Phenomena 1973, 2, 33.

5. Byberg, J. R.; Spanget-Larsen, J. Chem Phys Lett 1973, 23, 247.

6. Linderberg, J.; Öhrn, Y.; Thulstrup, P. W. in Quantum Science; Calais, J. L.; Goscinski, O.; Linderberg, J.; Öhrn, Y., Eds.; Plenum: New York, 1976; pp. 93-103.

7. Dalgård, E.; Linderberg, J. Int J Quantum Chem Symp 1975, 9, 269.

8. Dalgård, E.; Linderberg, J. J Chem Phys 1976, 65, 692.

9. Deshmukh, P.; Linderberg, J. Int J Quantum Chem 1981, 19, 575.

10. Wolfsberg, M.; Helmholtz, L. J Chem Phys 1952, 20, 837.

11. Lohr, L. L.; Lipscomb, W. N. J Chem Phys 1963, 38, 1607; Hoffmann, R. J Chem Phys 1963, 39, 1397.

12. Zerner, M. C. Rev Comp Chem 1991, 2, 313.

13. Porezag, D.; Frauenheim, Th.; Köhler, Th.; Seifert, G.; Kaschner, R. Phys Rev B 1995, 51, 12947; Seifert, G.; Porezag, D.; Frauenheim, Th. Int J Quantum Chem 1996, 58, 185-192.

14. Gross, A.; Scheffler, M.; Mehl, M. J.; Papaconstantopoulos, D. A. Phys Rev Lett 1999, 82, 1209, and references therein.

15. Lindholm, E.; Lundqvist, S. Phys Scripta 1985, 32, 220.

16. Lindholm, E.; Åsbrink, L. Molecular Orbitals and Their Energies, Studied by the Semiempirical HAM Method; Springer: New York, 1985.

17. Stowasser, R.; Hoffmann, R. J Am Chem Soc 1999, 121, 3414.

18. Kohn, W.; Becke, A. D.; Parr, R. G. J Phys Chem 1996, 100, 12974.

19. Harris, J. Phys Rev B 1985, 31, 1770.

20. Foulkes, W. M. C.; Haydock, R. Phys Rev B 1989, 39, 12520.

21. Freed, K. F. in Structure and Dynamics of Atoms and Molecules: Conceptual Trends; Calais, J. L.; Kryachko, E., Eds.; Kluwer: Dordrecht, 1995; Freed, K. F. Acc Chem Res 1983, 16, 137.

22. Nicolas, G.; Durand, Ph. J Chem Phys 1979, 70, 2020; Sakai, Y.; Huzinaga, S. J Chem Phys 1982, 76, 2537. 